There was no positive impact over FP, or 498 over Placebo, for Diary Card symptoms, ROLQ or Nasal Airflow. Tmax occurred at approximately 5 hrs post dose, in keeping with previous studies. SB705498 was very well tolerated.

Conclusions In a robust clinical model of allergic rhinitis, there was no intrinsic activity demonstrated by SB705498 and no additive effect on a background of intranasal steroids. FP was highly effective in this study. We conclude that despite engagement of the TRPV1 receptor there was no translation to clinical efficacy, suggesting redundancy in symptom pathways.

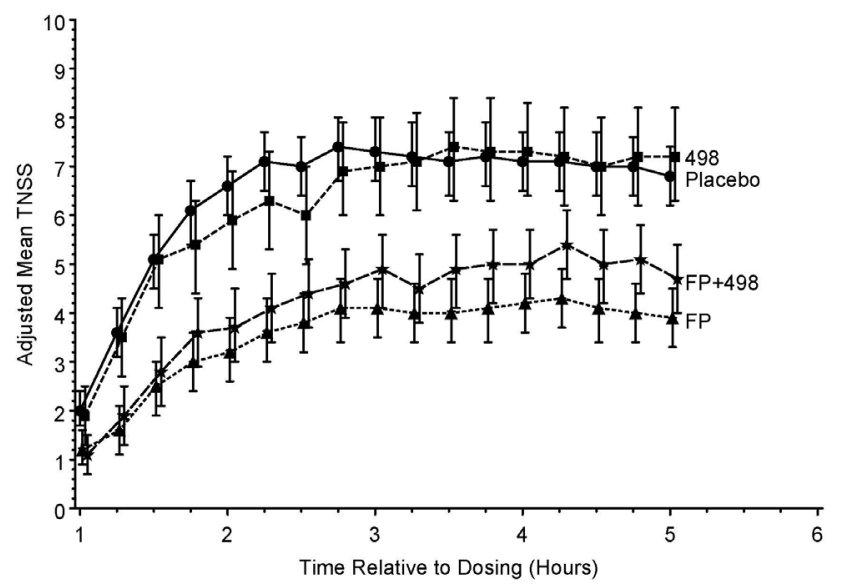

Abstract P153 Figure 1

\section{P154 TRPV1 IS NOT A TARGET FOR THE TREATMENT OF NON- ALLERGIC RHINITIS: A CLINICAL STUDY}

doi:10.1136/thoraxjnl-2012-202678.215

'RD Murdoch, 'P Bareille, 'J Bentley, 'A Newlands, ' $\mathrm{K}$ Smart, ${ }^{2} \mathrm{D}$ Patel. ' $\mathrm{G}$ laxoSmithKline, Stevenage, UK; ${ }^{2}$ Cetero Research, Mississauga, Canada

Background TRPV1 is a ligand gated ion channel activated by a range of physiological factors such at Temperature, $\mathrm{pH}$, and osmotic stress. In the nose, the TRPV1-expressing sensory c-fibres are thought to play a key role in the development of nasal hyperresponsiveness resulting in symptoms in NAR patients. We hypothesise that topical antagonism with a selective TRPV1 antagonist would offer substantial symptom control. SB705498 is a potent selective TRPV1 antagonist in animal and human models.

Methods $40 \mathrm{M} \& \mathrm{~F}$ NAR patients were enrolled into a randomised, double-blind, placebo controlled, 2 period crossover study of either $12 \mathrm{mg}$ SB705498 i.n. or placebo for 14 days with a 4 week washout. The study was conducted in a validated Environmental Challenge Chamber (Cetero) where patients were exposed to Cold Dry Air (CDA) at 14c, 15\% RH, air speed 5 feet/sec. Exposure was over the winter months in Canada. Diary card symptoms were analysed during home dosing on days when the temp was less than 14c. Two chamber sessions of $1 \mathrm{hr}$ duration, as well as medical history and neg skin prick testing, at screening were performed to establish a consistent diagnosis with a single challenge on Day $1+1 \mathrm{hr}$, Day $14+1 \mathrm{hr}$ and Day $14+24$ hr to establish the drug response. Post dose TSS (rhinorrhoea, congestion, PND), sneezing and ocular symptoms were recorded, as was acoustic rhinomanometry, ROLQ, PK and Safety monitoring.

Results The primary outcome of weighted mean TSS over the challenge period or the maximum TSS was not impacted by administration of SB705498 relative to placebo (see figure). There was no impact on sneezing, ocular symptoms, acoustic rhinimanometry, or ROLO Compared with placebo, repeated doses of SB705498 did not alleviate TSS triggered by cold in a multistimuli wild type setting.
PK analysis supported an o.d. regimen with 2 fold accumulation over the dosing period.

Conclusions In a robust clinical model of non-allergic rhinitis, intranasal SB705498 12mg o.d. for 14 days did not alleviate the symptoms of NAR triggered by the most common provocation agent: Cold Dry Air. We conclude that despite engagement of the TRPV1 receptor there was no translation to clinical efficacy, suggesting redundancy in symptom pathways.

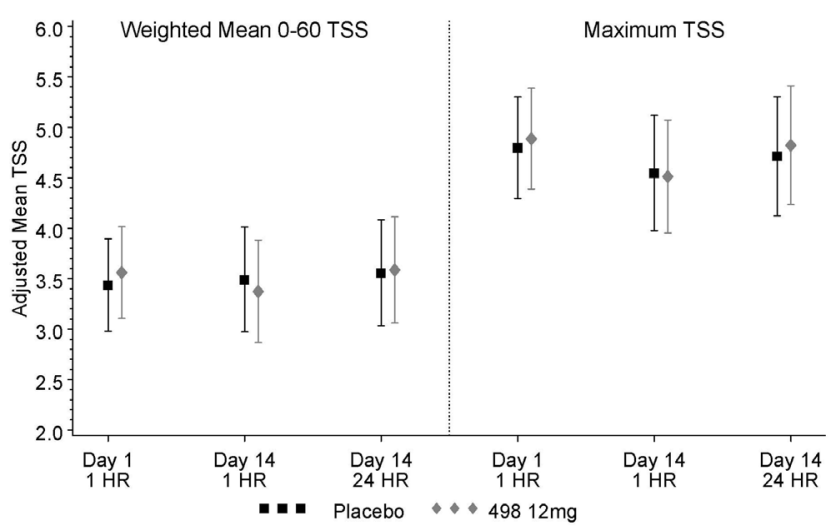

Abstract P154 Figure 1

\section{P155 VISUALISATION OF AIRWAY NERVES IN CHRONIC COUGH: TOWARDS THE IDENTIFICATION OF THE HUMAN 'COUGH RECEPTOR'}

doi:10.1136/thoraxjnl-2012-202678.216

'PW West, ${ }^{2 B J}$ Canning, 'EC Hilton, 'S Khalid, 'K Holt, 'R Abdulqawi, 'AA Woodcock, 'JA Smith. 'University of Manchester, Manchester, United Kingdom; ' $J o h n s ~ H o p k i n s$ Asthma \& Allergy Centre, Baltimore, USA

Introduction The spinal and vagal innervation of the respiratory tract is well defined, particularly in animals. These discoveries include the identification of pathways involved in provoking cough and descriptions of the guinea pig 'cough receptor'. However, many of the immunochemical features of the airway afferents described in animals have yet to be defined in humans, yet plasticity of these airway afferents may be important in the pathophysiology of chronic cough.

Objectives To define and characterise the innervation present in bronchoscopic biopsies from patients with chronic cough. We aimed to carry out the first ever whole mount immunohistochemical studies of airway nerves in cough patients, a technique that should improve visualisation of these neuronal structures and their sites of termination.

Methods Biopsy tissue was gifted from patients undergoing clinical investigation for chronic cough. Tissue, sampled from throughout the extrapulmonary airways, was immediately fixed in $4 \%$ paraformaldehyde. Non-specific antibody binding was blocked using $10 \%$ normal serum and $1 \%$ skimmed milk powder, diluted in PBS, before application of primary antibodies. Polyclonal rabbit anti-PGP9.5 (Ultraclone, UK) and monoclonal mouse anti-neurofilament (NF200, Leica Biosystems, UK) were applied at a dilution of 1:1000 and 1:200 respectively. Primary antibody binding was detected using appropriate Alexa-fluor conjugated secondary antibodies and whole mount preparations were visualised using epifluorescence and confocal microscopy. Images of biopsy staining were subject to morphometric analysis.

Results Many epithelial, subepithelial and intramuscular fibres were detected using both antibodies. Co-staining revealed that only PGP9.5 defined pulmonary neuroendocrine cells and better elucidated varicose epithelial fibres. A proportion of all fibre types were 